(C) 2010, IEEE. Reprinted, with permission, fromSanchez-Curto, J, Chamorro-Posada, P and McDonald, G, Refraction of grey solitons at defocusing Kerr interfaces, Advanced Optoelectronics and Lasers (CAOL), 2010 International Conference, 2010.

This material is posted here with permission of the IEEE. Such permission of the IEEE does not in any way imply IEEE endorsement of any of the University of Salford's products or services. Internal or personal use of this material is permitted. However, permission to reprint/republish this material for advertising or promotional purposes or for creating new collective works for resale or redistribution must be obtained from the IEEE by writing to pubs-permissions@ieee.org. 


\title{
Refraction of grey solitons at defocusing Kerr interfaces
}

\author{
Julio Sánchez-Curto, ${ }^{1}$ Pedro Chamorro-Posada, ${ }^{1}$ and Graham S. McDonald ${ }^{2}$ \\ ${ }^{1}$ Departamento de Teoría de la Señal y Comunicaciones e Ingeniería Telemática, \\ Universidad de Valladolid, ETSI Telecomunicación, Paseo Belén 15, Valladolid 47011, Spain \\ ${ }^{2}$ Joule Physics Laboratory, School of Computing, Science and Engineering, \\ University of Salford, Salford M5 4WT, United Kingdom
}

\begin{abstract}
We present a generalized Snell's law that governs grey soliton refraction at the interface separating two defocusing Kerr media. The analysis, based on the Helmholtz theory, is valid for arbitrary angles of incidence and reveals that grey solitons undergo either external or internal refraction depending on the soliton contrast parameter.
\end{abstract}

\section{INTRODUCTION}

Nonlinear interfaces play a pivotal role in nonlinear science. The theory ruling the behaviour of spatial solitons at nonlinear interfaces is the cornerstone for the explanation of more complex phenomena such as the propagation in nonlinear waveguides [1], directional couplers [2] or all-optical gates [3], [4]. A vast literature on the subject lasts for three decades, since the pioneering works devoted to linear/nonlinear interfaces [5], [6], [7] till the recent studies on interfaces separating quadratic [8] or photorefractive [9] media. Those phenomena associated with nonlinear interfaces such as the formation of nonlinear surface waves [6], [10], [11], the emission of multisoliton patterns [12], [13], [14] and the existence of giant Goos Hänchen shift [15] have been analyzed in a large variety of media and interface configurations. Besides the theoretical works, experiments on nonlinear interfaces have been also carried out to verify analytical predictions [16], [17].

A review of the literature on nonlinear interfaces may conclude that two main features are found in a large number of works. First, most studies have relied on the Nonlinear Schrödinger (NLS) equation to describe soliton evolution in nonlinear media. Since the paraxial approximation is assumed in the NLS, the validity of the analysis is limited to vanishingly small angles of incidence or refraction. Such is the case, for instance, of the successful particle-like model of Aceves et al. [18], [19] where the complicated dynamical evolution of a beam at nonlinear interfaces is described by a simple Newtonian model. Secondly, the study of nonlinear interfaces has focused on bright solitons, so that the analysis of dark solitons has been only considered in a few number of works [20], [21].

Nonlinear interfaces have an inherent nonparaxial character which manifests, for instance, when a soliton acquires a large angle of propagation due to the phenomenon of external refraction induced by the nonlinear interface. This sort of nonparaxiality, associated to the propagation of broad beams (in relation to its wavelength) with large angles in relation to the reference axes, is properly described in the framework of the Helmholtz theory [22], [23]. Unlike the NLS, the Nonlinear Helmholtz (NLH) equation [24], [22] preserves rotations and removes previous angular limitations. The analysis of nonlinear interfaces based on the Helmholtz theory thus collects the full angular content of the problem which is summarized in a generalized Snell's law [25], [26]. Initially reported for addressing bright soliton refraction at the interface separating two focusing Kerr media [25], it has been recently extended to govern soliton refraction in defocusing Kerr media [27].

\section{GENERALIZED SNELL'S LAW FOR GREY SOLITONS}

Our analysis of grey soliton evolution at defocusing Kerr interfaces is based on the NLH equation

$$
\begin{array}{r}
\kappa \frac{\partial^{2} u}{\partial \zeta^{2}}+j \frac{\partial u}{\partial \zeta}+\frac{1}{2} \frac{\partial^{2} u}{\partial \xi^{2}}-|u|^{2} u= \\
{\left[\frac{\Delta}{4 \kappa}-(1-\alpha)|u|^{2}\right] H(\xi) u}
\end{array}
$$

whose derivation for a TE optical field satisfying a Helmholtz equation can be found in [26]. $u(\xi, \zeta)$ is the complex envelope of a forward propagating beam evolving along the normalized transverse $\xi=2^{1 / 2} x / w_{0}$ and longitudinal $\zeta=z / L_{D}$ coordinates. $w_{0}$ is the waist of a reference Gaussian beam with diffraction length $L_{D}=k w_{0}^{2} / 2$ and $\kappa=1 / k^{2} w_{0}^{2}$ is a nonparaxiality parameter relating the beam width in relation to the number of wavelengths $(\lambda=2 \pi / k)$ in the full width $2 w_{0}$ of a reference Gaussian beam [22], [23]. $\Delta=1-n_{02}^{2} / n_{01}^{2}$ and $\alpha=\alpha_{2} / \alpha_{1}$ account for the linear and nonlinear refractive index mismatch at the interface, respectively. As it is displayed at both sides of the interface shown in Fig. 1, we have assumed that the refractive index of the $i^{t h}$ medium is $n_{i}=n_{0 i}-\alpha_{i} I$ where $n_{0 i}$ is the linear refractive index, $\alpha_{i}$ stands for the defocusing Kerr coefficient and $I$ is the optical intensity.

Matching the phase of the solution solutions for focusing [22], [23], [28] and defocusing [29] Kerr media at both sides of the discontinuity one obtains a nonlinear Snell's law [25], [26], [27]

$$
\gamma_{ \pm} n_{01} \cos \left(\theta_{n i}+\theta_{0 i}\right)=n_{02} \cos \left(\theta_{n t}+\theta_{0 t}\right) .
$$


where $\theta_{n i}$ and $\theta_{n t}$ are the net angles of incidence and refraction of a grey soliton, respectively. They account for the total angle between the propagation direction of the solitor dip and the interface as it is illustrated by the grey arrows ir Fig. 1. The interface separating two defocusing Kerr media is represented by a dotted line and has been rotated in relatior to the normalized coordinates.

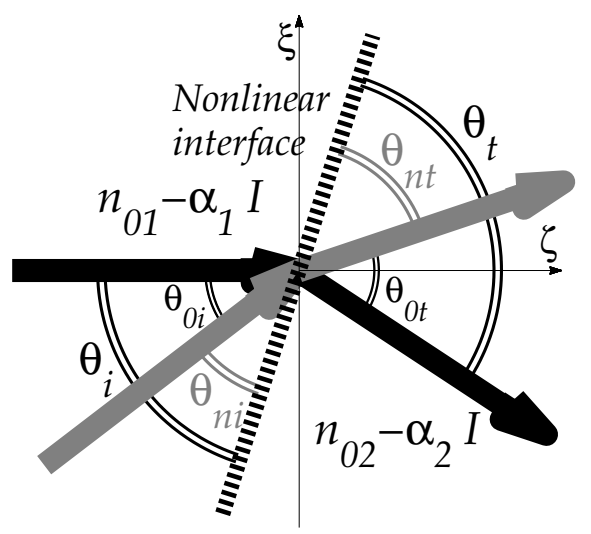

Fig. 1. Angular relationships involved in black and grey soliton refraction.

$\theta_{0 i}$ and $\theta_{0 t}$ represent the intrinsic angles of the incident [29] and refracted grey soliton [27] relative to the propagation direction of the background wave supporting the corresponding dark soliton. Their values are deduced from the condition supplementing Eq. (2), i.e. the preservation of the soliton grayness parameter at both sides of the interface [27]. $\theta_{0 i}$ and $\theta_{0 t}$ make net angles of incidence and refraction of the grey soliton differ from the ones associated to the background wave or the corresponding black soliton $\theta_{i}$ and $\theta_{t}$, respectively. Of course, the case of bright [25], [26] and black [27] soliton refraction can be deduced from Eq. (2) provided $\theta_{0 i}=\theta_{0 t}=0$.

In Eq. (2) $\gamma_{ \pm}$is a nonlinear correction term which has been previously calculated for bright $\left(\gamma_{+}\right)$[25] and black $\left(\gamma_{-}\right)$[27] solitons. In this case,

$$
\gamma_{-}=\left[\frac{\left(1-4 \kappa u_{0}^{2}\right)}{1-4 \kappa u_{0}^{2} \alpha(1-\Delta)^{-1}}\right]^{1 / 2}
$$

where $u_{0}$ denote the amplitude of the background wave supporting the soliton.

The nonparaxiality parameter $\kappa$ has been shown to play a pivotal role in the study of bright and black soliton refraction at nonlinear interfaces [25], [26]. The case of grey solitons is not an exception as the results shown in Fig. 2 reveal. Eq. (2) is represented for different values of the soliton contrast parameter $F$ [29], [27] when two values of $\kappa$ are used, i.e. $\kappa=10^{-4}$ (a) and $\kappa=10^{-3}$ (b). We assume a dark soliton $u_{0}=1$ impinging a nonlinear interface with $\alpha_{2}=4 \alpha_{1}$ and $n_{02}=1.0124 n_{01}$ Two different scenarios are found depending solely on $\kappa$. When $\kappa=10^{-4}, \gamma_{-} \approx 1$ and the net angle of refraction is dictated in Eq. (2) by the relationship between the linear refractive indexes. Regardless of the value of the soliton (a)

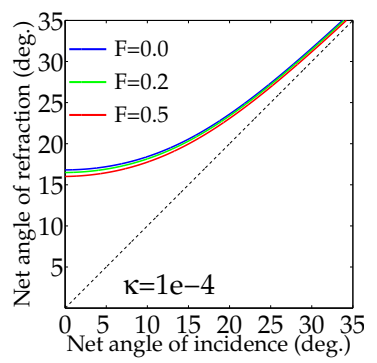

(b)

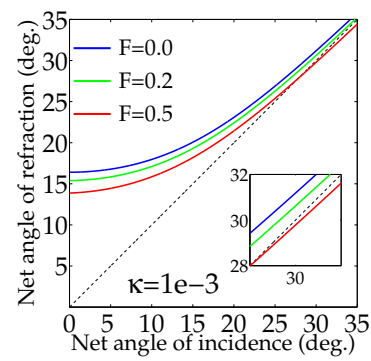

Fig. 2. Generalized Snell's law for a nonlinear interface with $\alpha_{2}=4 \alpha_{1}$ and $n_{02}=1.0124 n_{01}$ for $\kappa=10^{-4}$ (a) and $\kappa=10^{-3}$ (b).

greyness, all soliton will undergo a very similar net angle of refraction as it is shown in Fig. 2(a). This result changes when $\kappa=10^{-3}$ as Fig. 2(b) reveals. In this case $\gamma_{-} \approx n_{02} / n_{01}$, so that the net angle of refraction is dependent on the intrinsic angles of the incident and refracted solitons $\theta_{0 i}$ and $\theta_{0 t}$. The curve for $F=0.5$ shows a novel feature not previously found in black or bright soliton refraction. Unlike black solitons, which experience either external or internal refraction, grey solitons may undergo both types of refraction when the net angle of incidence changes.

The inset of Fig. 2(b) also reveals that grey soliton refraction depends on $F$. For a fixed net angle of incidence $\theta_{n i}=30^{\circ}$, the grey soliton with $F=0.2$ undergoes external refraction while internal refraction is achieved when $F=0.5$. This conclusion is in excellent agreement with the results extracted from numerical simulations shown in Fig. 3. The two snapshots in Fig. 3(a) and (b) are obtained from the numerical integration of the NLH [30] and correspond to those interface and soliton parameters used in Fig. 2(b). (a)

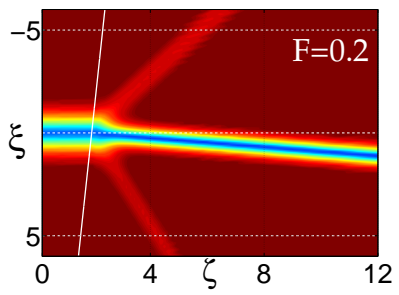

(b)

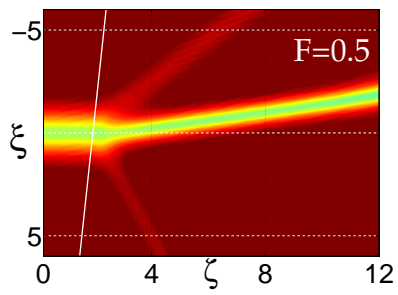

Fig. 3. Change from external (a) to internal (b) refraction when $F$ increases. In both cases, $\alpha_{2}=4 \alpha_{1}, n_{02}=1.0124 n_{01}$ and $\kappa=10^{-3}$.

A full description of those properties associated to the evolution of grey solitons at defocusing Kerr interfaces will be presented at the conference. Massive numerical simulations have been carried out in order to contrast the validity of the theoretical predictions.

\section{CONCLUSION}

In this work we have demonstrated the validity of the Helmholtz Snell's law to address not only bright or black, but also grey soliton refraction at the interface separating two Kerr media. Deduced in the framework of the Helmholtz 
theory, this Snell's law is valid for all possible values of any of the angles involved in contrast to the angular restrictions inherent in the paraxial theory. We have showed that a change from external to internal refraction is allowed in grey soliton refraction dependent on the angle of incidence.

\section{ACKNOWLEDGMENT}

The authors would like to thank the support provided by the Spanish Ministerio de Educación y Ciencia and Fondo Europeo de Desarrollo Regional, project TEC2007-67429C02-01, and Junta de Castilla y León, project VA001A08.

\section{REFERENCES}

[1] D. Mihalache, M. Bertolotti, and C. Sibilia, Nonlinear Wave Propagation in Planar Structures, ser. Progress in Optics XXVII. Elsevier Science Publishers, 1989.

[2] A.B. Aceves, J.V. Moloney, and A.C. Newell, "Theory of light-beam propagation at nonlinear interfaces. II. Multiple-particle and multipleinterface extensions," Physical Review A, vol. 39, no. 4, pp. 1828-1840, 1989.

[3] J. Scheuer and M. Orenstein, "Interactions and switching of spatial soliton pairs in the vicinity of a nonlinear interface," Optics Letters, vol. 24, no. 23, pp. 1735-1737, December 1999.

[4] — , "All-optical gates facilitated by soliton interactions in a multilayered Kerr medium,' Journal of the Optical Society of America B, vol. 22, no. 6, pp. 1260-1267, June 2005.

[5] A.E. Kaplan, "Theory of hysteresis reflection and refraction of light by a boundary of a nonlinear medium," Sov. Phys. JETP, vol. 45, no. 5, pp. 896-905, May 1977.

[6] W.J. Tomlinson, "Surface wave at a nonlinear interface," Optics Letters, vol. 5, no. 7, pp. 323-325, July 1980.

[7] W.J. Tomlinson, J.P. Gordon, P.W. Smith, and A.E. Kaplan, "Reflection of a gaussian beam at a nonlinear interface," Applied Optics, vol. 21, no. 11, pp. 2041-2051, June 1982.

[8] I.V. Shadrivov and A.A. Zharov, "Dynamics of optical spatial solitons near the interface between two quadratically nonlinear media," Journal of the Optical Society of America B, vol. 19, no. 3, pp. 596-602, March 2002.

[9] A.D. Boardman, P. Bontemps, W. Ilecki, and A.A. Zharov, "Theoretical demonstration of beam scanning and switching using spatial solitons in a photorefractive crystal," Journal of Modern Optics, vol. 47, no. 11, pp. 1941-1957, 2000.

[10] P. Varatharajah, A.B. Aceves, J.V. Moloney, and E.M. Wright, "Stationary nonlinear surface waves and their stability in diffusive Kerr-like nonlinear media," Journal of the Optical Society of America B, vol. 7, no. 2, pp. 220-229, February 1990.

[11] Y.V. Kartasov, F. Ye, V.A. Vysloukh, and L. Torner, "Surface waves in defocusing thermal media," Optics Letters, vol. 32, no. 15, pp. 2260 2262, August 2007.

[12] N.N. Rozanov, "Nonlinear reflections and transmission of limited light beams," Opt. Spectrosc., vol. 47, pp. 335-337, September 1979.

[13] E.M. Wright, G.I. Stegeman, C.T. Seaton, J.V. Moloney, and A.D. Boardman, "Multisoliton emission from a nonlinear waveguide," Physical Review A, vol. 34, no. 5, pp. 4442-4444, November 1986.

[14] Y.M. Aliev, A.D. Boardman, K. Xie, and A.A. Zharov, "Conserved energy approximation to wave scattering by a nonlinear interface," Physical Review E, vol. 49, no. 2, pp. 1624-1633, February 1994.

[15] M. Peccianti, G. Assanto, A. Dyadyusha, and M. Kaczmarek, "Nonlinear shift of spatial solitons at a graded dielectric interface," Optics Letters, vol. 32, no. 3, pp. 271-273, February 2007.

[16] O. Emile, T. Galstyan, A. Le Floch, and F. Bretenaker, "Measurement of the nonlinear Goos-Hänchen effect for gaussian optical beams," Physical Review Letters, vol. 75, no. 8, pp. 1511-1513, August 1995.

[17] E. Alvarado-Méndez, R. Rojas-Laguna, J.G. Aviña Fernández, M. Torres-Cisneros, J.A. Andrade-Lucio, J.C. Pedraza-Ortega, E.A. Kuzin, J.J. Sánchez-Mondragón, and V. Vysloukh, "Total internal reflection of spatial solitons at interface formed by a nonlinear saturable and a linear medium," Optics Communications, vol. 193, pp. 267-276, June 2001.
[18] A.B. Aceves, J.V. Moloney, and A.C. Newell, "Reflection, transmission, and stability characteristics of optical beams incident upon nonlinear dielectric interfaces," Journal of the Optical Society of America B, vol. 5, no. 2, pp. 559-564, February 1988.

[19] _ "Reflection and transmission of self-focused channels at nonlinear dielectric interfaces," Optics Letters, vol. 13, no. 11, pp. 1002-1004, November 1988.

[20] S.R. Skinner and D.R. Andersen, "Stationary fundamental dark surface waves," Journal of the Optical Society of America B, vol. 8, no. 4, pp. 759-764, April 1991.

[21] Y. Chen, "Bright and dark surface waves at a nonlinear interface," Physical Review A, vol. 45, no. 7, pp. 4974-4978, April 1992.

[22] P. Chamorro-Posada, G.S. McDonald, and G. New, "Nonparaxial solitons," Journal of Modern Optics, vol. 45, no. 6, pp. 1111-1121, 1998.

[23] - "Propagation properties of non-paraxial spatial solitons," Journal of Modern Optics, vol. 47, no. 11, pp. 1877-1886, 2000.

[24] G. Fibich, "Small beam nonparaxiality arrests self-focusing of optical beams," Physical Review Letters, vol. 76, no. 23, pp. 4356-4359, June 1996.

[25] J. Sánchez-Curto, P. Chamorro-Posada, and G.S. McDonald, "Helmholtz solitons at nonlinear interfaces," Optics Letters, vol. 32, no. 19, pp. 1126-1128, May 2007.

[26] — "Nonlinear interfaces: intrinsically nonparaxial regimes and effects," Journal of Optics A: Pure and Applied Optics, vol. 11, p. 054015, 2009.

[27] - "Dark solitons at nonlinear interfaces," Optics Letters, vol. 35, no. 9, pp. 1347-1349, April 2010.

[28] P. Chamorro-Posada, G.S. McDonald, and G. New, "Exact soliton solutions of the nonlinear Helmholtz equation," Journal of the Optical Society of America B, vol. 19, no. 5, pp. 1216-1217, 2002.

[29] P. Chamorro-Posada and G.S. McDonald, "Helmholtz dark solitons," Optics Letters, vol. 28, no. 10, pp. 825-827, 2003.

[30] P. Chamorro-Posada, G.S. McDonald, and G. New, "Non-paraxial beam propagation methods," Optics Communications, vol. 192, pp. 1-12, 2001. 